\title{
An A/G polymorphism rs3746444 in miR-499 is associated with increased cancer risk: a meta-analysis
}

\author{
N. Wang1, ZQ Tian' ${ }^{2}$ Y. Li ${ }^{1}$, R.M. Zhou' ${ }^{1}$ and G.Y. Wang ${ }^{3}$ \\ ${ }^{1}$ Department of Molecular Biology, \\ The Fourth Hospital of Hebei Medical University, Shijiazhuang, \\ Hebei, China \\ ${ }^{2}$ Department of Thoracic Surgery, \\ The Fourth Hospital of Hebei Medical University, Shijiazhuang, \\ Hebei, China \\ ${ }^{3}$ Second Department of General Surgery, \\ The Fourth Hospital of Hebei Medical University, Shijiazhuang, \\ Hebei, China \\ Corresponding author: G.-Y. Wang \\ E-mail: wangguiying_wgy@126.com / tizq12@vip.163.com
}

Genet. Mol. Res. 12 (3): 3955-3964 (2013)

Received April 9, 2013

Accepted August 20, 2013

Published September 23, 2013

DOI http://dx.doi.org/10.4238/2013.September.23.14

\begin{abstract}
An A/G polymorphism (rs3746444) has been identified in the miR-499 gene that can change the conformation of the secondary gene structure and thereby directly affect binding to target mRNAs and the microRNA (miRNA) maturation process, thus altering protein expression and potentially contributing to cancer susceptibility. Numerous studies investigating the association between the rs3746444 polymorphism and cancers have been published; however, results are inconsistent and inconclusive. To
\end{abstract}


clarify the relationship between the miR-499 rs3746444 polymorphism and cancer, we conducted a comprehensive meta-analysis on 14 case-control studies comprising 7189 cases and 8577 controls. Odds ratios (OR) and 95\% confidence intervals (CI) were calculated by using dominant, recessive, and co-dominant genetic models. A publication bias test and subgroup analysis were also performed. Results showed that the $\mathrm{G}$ allele was associated with a significantly increased cancer risk compared to the A allele $(\mathrm{OR}=1.09 ; 95 \% \mathrm{CI}=$ 1.00-1.18). Similarly, moderately elevated risks were also observed in overall analyses in the dominant model $(\mathrm{OR}=1.13 ; 95 \% \mathrm{CI}=$ 1.01-1.26). Moreover, significantly increased risks were observed in Asian populations $(\mathrm{G}$ allele $v s$ A allele: $\mathrm{OR}=1.18 ; 95 \% \mathrm{CI}=$ 1.01-1.37; GG vs AA: $\mathrm{OR}=1.36 ; 95 \% \mathrm{CI}=1.07-1.73$; dominant model: $\mathrm{OR}=1.19 ; 95 \% \mathrm{CI}=1.00-1.41$; recessive model: $\mathrm{OR}=$ $1.31 ; 95 \% \mathrm{CI}=1.03-1.66$ ), but not in European populations. These findings indicate that the miR-499 rs3746444 polymorphism is associated with an increased cancer risk.

Key words: MiR-499; Meta-analysis; Polymorphism; Cancer

\section{INTRODUCTION}

MicroRNAs (miRNAs) are an abundant class of small, noncoding, single-stranded RNAs of 21 to 24 nucleotides that form base pairs with target mRNAs and regulate their posttranscriptional functions as tumor suppressors and oncogenes (Bartel, 2004; Esquela-Kerscher and Slack, 2006; Vasudevan et al., 2007). Many studies in humans have provided evidence that the presence of single nucleotide polymorphisms (SNPs) in miRNAs can alter miRNA processing, expression, and/or binding to target mRNAs, thereby representing another type of genetic variability that can contribute to susceptibility to cancer development (Zeng and Cullen, 2003; Loktionov, 2004; Duan et al., 2007).

An A/G polymorphism (rs3746444) has been identified in the miR-499 gene. This polymorphism is located in the stem region opposite the mature miR-499 sequence, and it results in a change from an A:U pair to a $\mathrm{G}: \mathrm{U}$ mismatch in the stem structure of miR499 (Hu et al., 2008). The optimal free energy was decreased from $-62.30 \mathrm{kcal} / \mathrm{mol}$ for A to $-61.90 \mathrm{kcal} / \mathrm{mol}$ for $\mathrm{G}$ alleles, suggesting a less stable secondary structure of miR-499 with the $G$ allele compared to the A allele. Genetic variants in mature miRNA regions were shown to change the conformation of the secondary structure and thereby directly affect both the binding to target mRNAs and the miRNA maturation process (Zeng and Cullen, 2003; Duan et al., 2007), thus altering protein expression and contributing to cancer susceptibility (Bartels et al., 2009).

To date, many molecular epidemiological studies have been performed to evaluate the role of the rs 3746444 polymorphism on various cancers such as breast, liver, lung, gastric, colorectal, prostate, etc. (Tian et al., 2009; Hu et al., 2009; Liu et al., 2010; Okubo et al., 2010; Catucci et al., 2010; Srivastava et al., 2010; Akkiz et al., 2011; George et al., 2011; Mittal et al., 2011; Vinci et al., 2011; Min et al., 2012; Xiang et al., 
2012; Zhou et al., 2011, 2012). The frequency of the G allele varies in different geographic areas and ethnic populations. Moreover, genetic effects of the polymorphism have been shown to vary from one type of cancer to the other. Even at the same tumor site, the results are conflicting. Consequently, the statistical power of an individual study could be very limited for efficient assessment of the rs3746444 polymorphism. Therefore, integration of these data sets may provide improved statistical power to detect any significant effects.

With the aim of addressing inconsistencies in the findings of these studies, we adopted a meta-analysis based on published case-control studies in an attempt to assess the association between the miR-499 rs3746444 polymorphism and cancer susceptibility.

\section{MATERIAL AND METHODS}

\section{Literature search strategy}

We searched MEDLINE (US National Library of Medicine, Bethesda, MD) for all genetic association studies of the rs3746444 polymorphism of miR-499 and cancer susceptibility published before April 2012 using the PubMed search engine. The search was limited to English-language papers, and the following keywords and subject terms were used: "miR-499" or "microRNA-499" or "miRNA-499", "A/G polymorphism" or "rs3746444", "polymorphism" or "SNPs", and "cancer and/or carcinoma and/or neoplasm". The references of all MEDLINE-identified publications were searched. In addition, the PubMed option "Related Articles" and publications on the same topic in the reference lists of the reviewed articles were retrieved to search for other potentially relevant publications. If an article reported results from different studies, each study was treated as a separate comparison in our meta-analysis.

\section{Selection criteria}

Any human associated study, regardless of sample size, was included if it met the following criteria: a) the study used an unrelated case-control design, b) the study investigated the association between rs3746444 polymorphisms of miR-499 and the risk of cancer, and c) the study was published in English. For articles using the same population resource or overlapping data sets, only the publication reporting the largest or most recent data set was included. Ultimately, data for this meta-analysis were available from 14 case-control studies, including 7189 cases with different types of tumors and 8577 controls

\section{Data extraction}

Two investigators independently extracted data and reached a consensus on all of the items. The following information was recorded for each study: first author, year of publication, country of origin, cancer type, ethnicity, number of cases and controls, study design, genotyping methods, and evidence of Hardy-Weinberg equilibrium (HWE). For subjects of different ethnicities, data were extracted separately and categorized as European or Asian (Table 1). 


\section{Statistical analysis}

Observed genotype frequencies for rs3746444 polymorphisms in controls were examined for deviations from HWE using a goodness-of-fit $\chi^{2}$-test with one degree of freedom. Odds ratios (ORs) were used as a measure of the association between the miR-499 rs3746444 polymorphism and risk of cancer. We evaluated the risk of genotypes AA and AG on cancers compared with that of the AA homozygote, and then calculated the ORs of GG+AG vs AA and $\mathrm{GG} v s \mathrm{AG}+\mathrm{AA}$ using dominant and recessive genetic models of the $\mathrm{G}$ allele, respectively. The statistical heterogeneity among studies was assessed with the $\chi^{2}$-based Q-test (Cochran, 1954) and $\mathrm{I}^{2}$ statistics (Higgins et al., 2003). When heterogeneity was not an issue, a fixed effect model was used with the Mantel-Haenszel method. Otherwise, a random effect model was used with the inverse variance method.

We examined the following study characteristics: cancer types (if one cancer type contained less than three studies, it was merged into the "other cancers" group), ethnicities, genotyping methods, study design (hospital-based studies and population-based studies), HWE, and quality score; the quality of each study was assessed according to Shen et al. (2012). Total scores ranged from 0 (worst) to 15 (best). Reports scoring $\leq 10$ were classified as "low quality", and those scoring $>10$ were classified as "high quality".

Publication bias was investigated with a funnel plot, which is the main graphical method of assessing bias. To supplement the funnel plot approach, the Begg and Mazumdar adjusted rank correlation test (Begg and Mazumdar, 1994) and the Egger regression asymmetry test (Egger et al., 1997) were adopted. To explore sources of heterogeneity across studies, we performed stratified and logistic meta-regression analyses.

All analyses were conducted with review manager software (RevMan version 5.0, The Cochrane Collaboration, Oxford, England) and STATA software (version 10.0, Stata Corporation, College Station, TX, USA). All P values were two-sided. Statistical tests performed in the present analysis were considered significant whenever the corresponding null-hypothesis probability was $\mathrm{P}<0.05$.

\section{RESULTS}

\section{Study characteristics}

Overall, 15 data sets extracted from 14 studies including 7189 cases and 8577 controls were available for this analysis. Study characteristics are summarized in Table 1. The sample size in these case-control studies varied considerably (range 200-2239 individuals). There were seven studies of Asian descendants and eight studies of European descendants. Several genotyping methods were used, including TaqMan, polymerase chain reaction-restriction fragment length polymorphism (PCR-RFLP), and high-resolution melting analysis (HRMA). Furthermore, approximately $80 \%(12 / 15)$ of these studies included descriptions of genotyping quality control measures, such as positive and negative controls, blindness to the case-control status, an independent genotyping assay to confirm the data, and/or random repetition of a portion of samples. The genotype distributions among the controls of all studies were consistent with HWE except for four studies (Okubo et al., 2010; Akkiz et al., 2011; Mittal et al., 2011; Zhou et al., 2011). 


\begin{tabular}{|c|c|c|c|c|c|c|c|c|}
\hline First author & Year & Country & Cancer type & Racial descent & Genotyping & Source of control & $\begin{array}{l}\text { Sample size } \\
\text { (case/control) }\end{array}$ & $\mathrm{P}^{\mathrm{a}}$ \\
\hline Akkiz (9) & 2011 & Turkey & Hepatocellular carcinoma & European & PCR-RFLP & Hospital-based & $222 / 222$ & 0.036 \\
\hline Catucci (10) & 2010 & Germany & Breast cancer & European & Taqman & Hospital-based & $823 / 925$ & 0.893 \\
\hline Catucci (10) & 2010 & Italy & Breast cancer & European & Taqman & Hospital-based & $756 / 1242$ & 0.250 \\
\hline George (11) & 2011 & India & Prostate cancer & European & PCR-RFLP & Hospital-based & $159 / 230$ & 0.073 \\
\hline $\mathrm{Hu}(12)$ & 2009 & China & Breast cancer & Asian & PCR-RFLP & Population-based & $1009 / 1093$ & 0.057 \\
\hline Liu (13) & 2010 & USA & SCCHN & European & PCR-RFLP & Hospital-based & $1109 / 1130$ & 0.441 \\
\hline $\operatorname{Min}(14)$ & 2012 & Korea & Colorectal cancer & Asian & PCR-RFLP & Population-based & $446 / 502$ & 0.453 \\
\hline Mittal (15) & 2011 & India & Bladder cancer & European & PCR-RFLP & Hospital-based & $212 / 250$ & 0.020 \\
\hline Okubo (16) & 2010 & Japan & Gastric cancer & Asian & PCR-RFLP & Hospital-based & $552 / 697$ & 0.048 \\
\hline Srivastava (17) & 2010 & India & Gallbladder cancer & European & PCR-RFLP & Population-based & $230 / 230$ & 0.566 \\
\hline Tian (18) & 2009 & China & Lung cancer & Asian & PCR-RFLP & Population-based & $1058 / 1035$ & 0.404 \\
\hline Vinci (19) & 2011 & Italy & Lung cancer & European & HRMA & Hospital-based & $101 / 129$ & 0.503 \\
\hline Xiang (20) & 2012 & China & Hepatocellular carcinoma & Asian & PCR-RFLP & Hospital-based & $100 / 100$ & 0.284 \\
\hline Zhou (21) & 2011 & China & $\mathrm{CSCC}$ & Asian & PCR-RFLP & Hospital-based & $226 / 309$ & 0.005 \\
\hline Zhou (22) & 2012 & China & Hepatocellular carcinoma & Asian & PCR-RFLP & Hospital-based & $186 / 483$ & 0.100 \\
\hline
\end{tabular}

PCR-RFLP $=$ polymerase chain reaction-restriction fragment length polymorphism; HRMA = high-resolution melting analysis; SCCHN = squamous cell carcinoma of the head and neck; CSCC = cervical squamous cell carcinoma; ${ }^{\mathrm{P}}$ value of Hardy-Weinberg equilibrium in controls.

\section{Quantitative synthesis}

There was a wide variation in the $G$ allele frequency of rs3746444 among different ethnicities, ranging from 0.13 in an Asian population to 0.58 in a European population. The mean frequency of the $\mathrm{G}$ allele for Asian populations was $0.18 \pm 0.05$, which was significantly lower that of European populations $(0.31 \pm 0.12, t=2.63, \mathrm{P}=0.02)$. The evaluations of the association of miR-499 rs3746444 with cancer risk are shown in Table 2. Overall, the G allele was associated with a significantly increased cancer risk compared with the A allele $(\mathrm{OR}=$ $1.09 ; 95 \%$ confidence interval (CI), 1.00-1.18). Similarly, moderately elevated risks were also observed in overall analyses of the dominant model $(\mathrm{OR}=1.13 ; 95 \% \mathrm{CI}=1.01-1.26)$ (Figure 1). Moreover, significantly increased risks were observed in Asian populations ( $\mathrm{G}$ allele $v s \mathrm{~A}$ allele: $\mathrm{OR}=1.18 ; 95 \% \mathrm{CI}=1.01-1.37$; $\mathrm{GG} v \mathrm{AA}: \mathrm{OR}=1.36 ; 95 \% \mathrm{CI}=1.07-1.73$; dominant model: $\mathrm{OR}=1.19 ; 95 \% \mathrm{CI}=1.00-1.41$; recessive model: $\mathrm{OR}=1.31 ; 95 \% \mathrm{CI}=1.03-1.66$ ), but not in European populations. When stratified separately by "genotyping", we found that the G allele, the GG genotype, and the dominant model all increased cancer risk in the PCRRFLP group. Subgroup analysis of "HWE" and "score" indicated that significantly increased risks were found among no-HWE studies and "score $\leq 10$ " studies. However, no significant association was found in stratified analyses by "cancer type" and "study design" in any of the comparison models tested.

\section{Evaluation of heterogeneity}

There was heterogeneity among studies in the overall comparisons and subgroup analyses. To explore sources of heterogeneity across studies, we assessed the allele comparison ( $\mathrm{G}$ allele $v s \mathrm{~A}$ allele), the heterozygote comparison (AG vs AA), and the dominant model comparison (GG+AG vs AA) by covariate "cancer type", "ethnicity", "genotyping methods", "study design", and "HWE". Results indicated that these covariates could not explain the sources of heterogeneity (Table 3). 


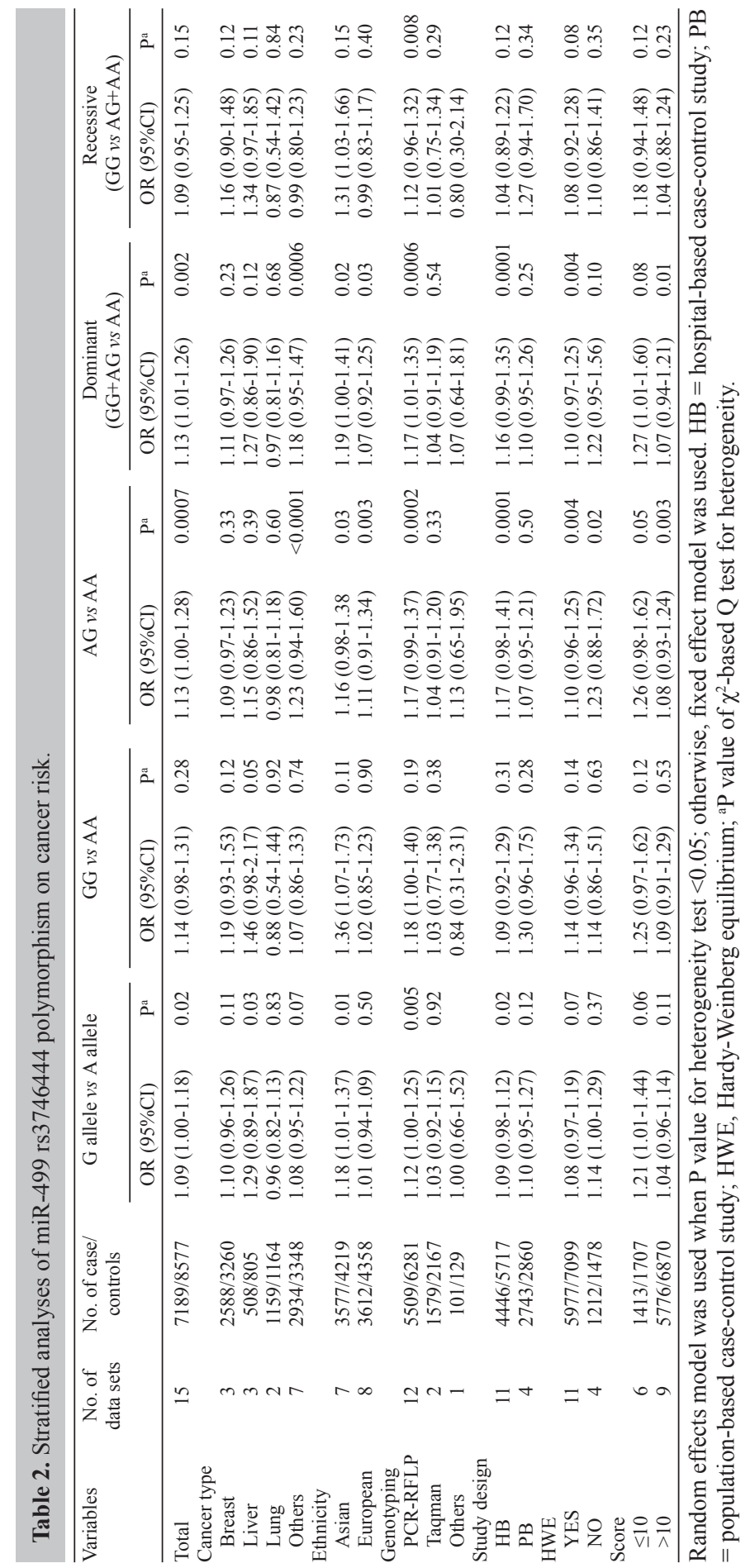




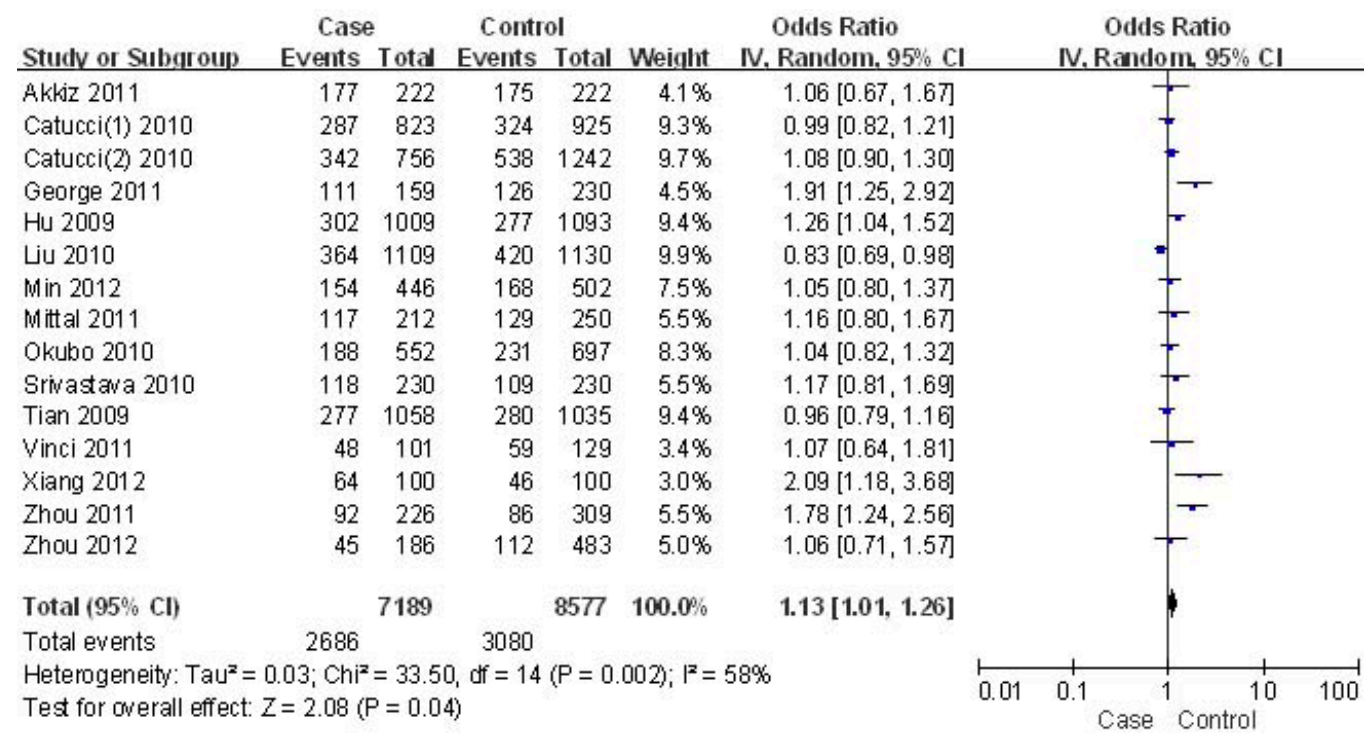

Figure 1. Forest plot of cancer risk associated with the GG+AG genotypes compared with the AA genotype in overall analyses (dominant model).

Table 3. Meta-regression analysis for exploring sources of heterogeneity.

\begin{tabular}{|c|c|c|c|c|c|c|c|c|c|c|c|c|}
\hline \multirow[t]{2}{*}{ Covariates } & \multicolumn{4}{|c|}{$\mathrm{G}$ allele $v s \mathrm{~A}$ allele } & \multicolumn{4}{|c|}{$\mathrm{AG} v s \mathrm{AA}$} & \multicolumn{4}{|c|}{ Dominant $(\mathrm{GG}+\mathrm{AG} v s \mathrm{AA})$} \\
\hline & $\begin{array}{l}\tau 2 \text { before } \\
\text { regression }\end{array}$ & $\begin{array}{l}\tau 2 \text { after } \\
\text { regression }\end{array}$ & $\begin{array}{l}\text { Adj R- } \\
\text { squared } \\
(\%)\end{array}$ & $P$ & $\begin{array}{l}\tau 2 \text { before } \\
\text { regression }\end{array}$ & $\begin{array}{l}\tau 2 \text { after } \\
\text { regression }\end{array}$ & $\begin{array}{c}\text { Adj R- } \\
\text { squared } \\
(\%)\end{array}$ & $P$ & $\begin{array}{l}\tau 2 \text { before } \\
\text { regression }\end{array}$ & $\begin{array}{l}\tau 2 \text { after } \\
\text { regression }\end{array}$ & $\begin{array}{c}\text { Adj R- } \\
\text { squared } \\
(\%)\end{array}$ & $\mathrm{P}$ \\
\hline Ethnicity 5 & 0.01028 & 0.00826 & 19.61 & 0.170 & 0.03903 & 0.04396 & -12.63 & 0.684 & 0.02630 & 0.02777 & -5.60 & 0.459 \\
\hline $\begin{array}{l}\text { Genotyping } \\
\text { methods } 6\end{array}$ & & 0.01169 & -13.69 & 0.469 & & 0.04407 & -12.91 & 0.657 & & 0.02985 & -13.50 & 0.549 \\
\hline Study design 7 & & 0.01224 & -19.05 & 0.907 & & 0.04613 & -18.19 & 0.620 & & 0.03216 & -22.29 & 0.743 \\
\hline Cancer type 4 & & 0.01096 & -6.66 & 0.667 & & 0.04798 & -22.93 & 0.643 & & 0.03292 & -25.19 & 0.840 \\
\hline HWE 2 & & 0.01075 & -4.55 & 0.544 & & 0.04081 & -4.57 & 0.539 & & 0.02708 & -2.98 & 0.526 \\
\hline Joint test & & 0.01427 & -38.78 & 0.775 & & 0.07609 & -94.95 & 0.970 & & 0.05212 & -98.17 & 0.944 \\
\hline
\end{tabular}

\section{Publication bias}

The Egger's test and the Begg's test were performed to evaluate the publication bias in the cancer literature. Figure 2 displays the funnel plot that was used to examine the association between the miR-499 rs3746444 polymorphism and overall cancer risk in the dominant model. For the allele comparison ( $\mathrm{G}$ allele $v s$ A allele), a marginally significant effect $(\mathrm{P}=0.048)$ was detected using Begg's test, but no significance was found using Egger's test. However, the bias disappeared when we excluded studies $9,15,16$, and 21 , which all showed deviations from HWE (Begg's test, $\mathrm{P}=0.119$ ). No evidence of publication bias was observed in the other comparison models. 


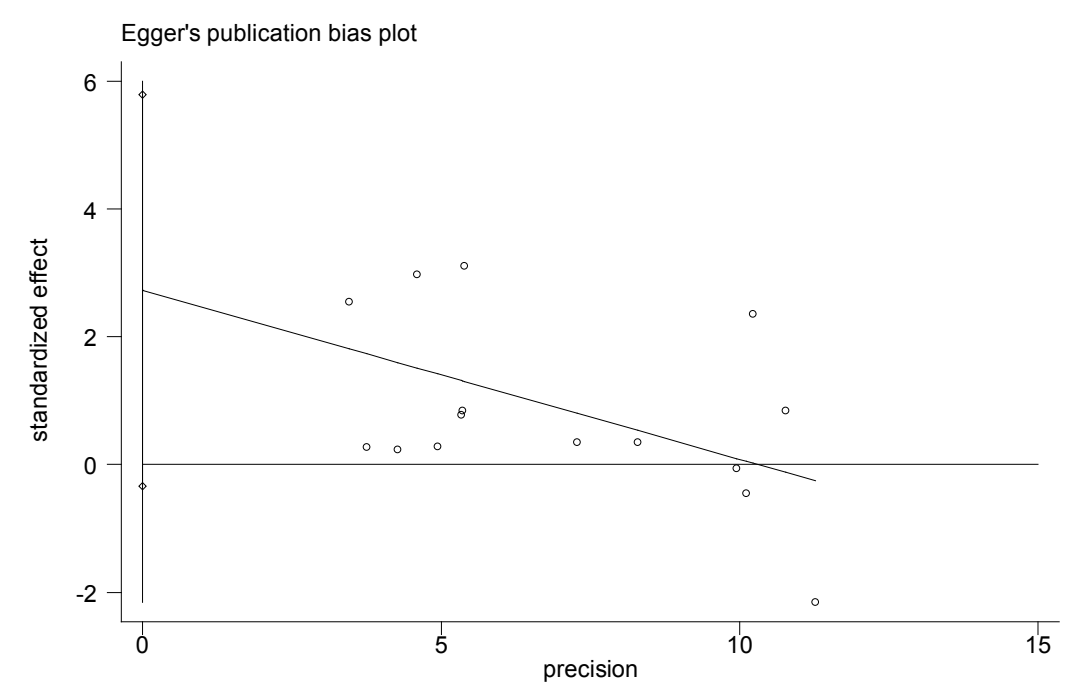

Figure 2. Egger's funnel plot for publication bias test (GG+AG vs AA, dominant model). Each point represents a separate study for the indicated association.

\section{DISCUSSION}

Many studies have demonstrated a relationship between the miR-499 rs3746444 polymorphism and the risk of cancer. However, the results were generally inconsistent (Hu et al., 2009; Liu et al., 2010; Xiang et al., 2012). Recently, meta-analysis has become a very powerful tool for combining results of various studies, enabling summarization of the main conclusions, and providing high statistical power for testing research hypotheses. Therefore, we conducted a meta-analysis to clarify the association between the miR-499 rs3746444 polymorphism with cancers.

To the best of our knowledge, this is the first meta-analysis of the association between the miR-499 rs3746444 polymorphism and cancer susceptibility. Our meta-analysis, which was based on 15 data sets extracted from 14 case-control studies with 7189 cases and 8577 controls, showed that the miR-499 rs3746444 G allele was associated with the risk of cancer, and that this association may vary when stratified for cancer type, ethnicity, genotyping method, and study design. The Hu et al. (2008) function test revealed that the $\mathrm{G}$ allele of rs 3746444 variants in the miR-499 region could decrease the stability of the secondary structure and consequently affect the miR-499 maturation process or binding to target mRNAs. This phenomenon has been observed for genetic risk factors in prostate cancer (George et al., 2011) and breast cancer (Hu et al., 2009), and may explain the genetic association of cancers. In addition, we carried out subgroup analyses for "ethnicity" and observed significantly elevated risks in all comparison models with the Asian group, but not in the European group, which might be explained by potentially different mechanisms underlying tumorigenesis in different populations. In a subgroup analysis of "genotyping methods", we observed a significantly increased risk within the PCR-RFLP group, but not in the TaqMan group. Because the TaqMan method is more precise than the PCR-RFLP method, and a limited number of studies were included in the TaqMan group, this result might reflect a selection bias, and should be interpreted with caution. 
The interpretation of results of meta-analyses is often plagued by significant heterogeneity. Lack of attention to this commonly occurring problem may cause misleading statistical inferences. To test the significance of heterogeneity, we carried out Cochran's Q test and calculated the $\mathrm{I}^{2}$ statistic, which describes the magnitude of heterogeneity across the constituent studies. The most noteworthy finding from our meta-analyses was the substantial heterogeneity, particularly among comparison models of alleles ( $\mathrm{G}$ allele $v s \mathrm{~A}$ allele) and the co-dominant (AG vs AA) and dominant models. To further explore the sources of heterogeneity, we carried out a meta-regression analysis and found that heterogeneity could not be explained by the covariates "ethnicity", "genotyping methods", "study design," "cancer type", or "HWE" in all comparison models (Table 3).

Another crucial question for meta-analysis is publication bias. To assess this problem, we determined the relationship between the OR estimates in a logarithmic scale and their corresponding standard errors across all constituent data sets. The results showed that no obvious publication bias was detected in this analysis. In fact, both the Begg and Mazumdar adjusted rank correlation analysis and the Egger regression asymmetry test revealed no correlation between the estimate of ORs and sample size.

Similar to other meta-analyses and systematic reviews, this study was subject to potential bias owing to systematic and random errors (Cook et al., 1997). First, only published studies were included in the meta-analysis, indicating that publication bias may exist even though no obvious publication bias was detected in this analysis. Second, the number of studies included in the meta-analysis was too small to perform a subgroup analysis for every type of cancer. Third, obvious heterogeneity was observed among the included studies, but the sources of this heterogeneity were not clear. Fourth, three studies showing genotype distributions of the control population that were not in HWE were included in this meta-analysis. Fifth, the lack of original data from the meta-analysis limited further evaluations of potential gene-gene and gene-environment interactions. Therefore, selection bias may be present, and we must draw conclusions with caution.

In conclusion, our meta-analysis suggested that the miR-499 rs3746444 polymorphism is associated with an increased cancer risk. Further stratification by ethnicity (Asians and Europeans) and genotyping method (PCR-RFLP and TaqMan) also identified a significant association of this polymorphism with cancer risk, especially in Asians and the "PCR-RFLP" group. To further evaluate the interactions between the rs3746444 polymorphisms and cancer risks, well-designed case-control studies with larger sample sizes are required.

\section{ACKNOWLEDGEMENTS}

Research supported by the Natural Science Foundation of Hebei Province, China (\#C2010000628).

\section{REFERENCES}

Akkiz H, Bayram S, Bekar A, Akgollu E, et al. (2011). Genetic variation in the microRNA-499 gene and hepatocellular carcinoma risk in a Turkish population: lack of any association in a case-control study. Asian Pac. J. Cancer Prev. 12: 3107-3112

Bartel DP (2004). MicroRNAs: genomics, biogenesis, mechanism, and function. Cell 116: 281-297.

Bartels CL and Tsongalis GJ (2009). MicroRNAs: novel biomarkers for human cancer. Clin. Chem. 55: 623-631. 
Begg CB and Mazumdar M (1994). Operating characteristics of a rank correlation test for publication bias. Biometrics 50: 1088-1101.

Catucci I, Yang R, Verderio P, Pizzamiglio S, et al. (2010). Evaluation of SNPs in miR-146a, miR196a2 and miR-499 as low-penetrance alleles in German and Italian familial breast cancer cases. Hum. Mutat. 31: E1052-E1057.

Cochran WG (1954). The combination of estimates from different experiments. Biometrics 10: 101-129.

Cook DJ, Mulrow CD and Haynes RB (1997). Systematic reviews: synthesis of best evidence for clinical decisions. Ann. Intern. Med. 126: 376-380.

Duan R, Pak C and Jin P (2007). Single nucleotide polymorphism associated with mature miR-125a alters the processing of pri-miRNA. Hum. Mol. Genet. 16: 1124-1131.

Egger M, Davey SG, Schneider M and Minder C (1997). Bias in meta-analysis detected by a simple, graphical test. BMJ 315: 629-634.

Esquela-Kerscher A and Slack FJ (2006). Oncomirs - microRNAs with a role in cancer. Nat. Rev. Cancer 6: 259-269.

George GP, Gangwar R, Mandal RK, Sankhwar SN, et al. (2011). Genetic variation in microRNA genes and prostate cancer risk in North Indian population. Mol. Biol. Rep. 38: 1609-1615.

Higgins JP, Thompson SG, Deeks JJ and Altman DG (2003). Measuring inconsistency in meta-analyses. BMJ 327: $557-$ 560.

Hu Z, Chen J, Tian T, Zhou X, et al. (2008). Genetic variants of miRNA sequences and non-small cell lung cancer survival. J. Clin. Invest. 118: 2600-2608.

Hu Z, Liang J, Wang Z, Tian T, et al. (2009). Common genetic variants in pre-microRNAs were associated with increased risk of breast cancer in Chinese women. Hum. Mutat. 30: 79-84.

Liu Z, Li G, Wei S, Niu J, et al. (2010). Genetic variants in selected pre-microRNA genes and the risk of squamous cell carcinoma of the head and neck. Cancer 116: 4753-4760.

Loktionov A (2004). Common gene polymorphisms, cancer progression and prognosis. Cancer Lett. 208: 1-33.

Min KT, Kim JW, Jeon YJ, Jang MJ, et al. (2012). Association of the miR-146aC $>$ G, 149C $>$ T, 196a2C $>$ T, and 499A $>$ G polymorphisms with colorectal cancer in the Korean population. Mol. Carcinog. 51 (Suppl 1): E65-E73.

Mittal RD, Gangwar R, George GP, Mittal T, et al. (2011). Investigative role of pre-microRNAs in bladder cancer patients: a case-control study in North India. DNA Cell Biol. 30: 401-406.

Okubo M, Tahara T, Shibata T, Yamashita H, et al. (2010). Association between common genetic variants in premicroRNAs and gastric cancer risk in Japanese population. Helicobacter 15: 524-531.

Shen SQ, Jiang DK, Liu GY, Chen F, et al. (2012). Meta-analysis shows significant association of the TP53 Arg72Pro with ovarian cancer risk. Mol. Biol. Rep. 39: 4683-4690.

Srivastava K, Srivastava A and Mittal B (2010). Common genetic variants in pre-microRNAs and risk of gallbladder cancer in North Indian population. J. Hum. Genet. 55: 495-499.

Tian T, Shu Y, Chen J, Hu Z, et al. (2009). A functional genetic variant in microRNA-196a2 is associated with increased susceptibility of lung cancer in Chinese. Cancer Epidemiol. Biomark. Prev. 18: 1183-1187.

Vasudevan S, Tong Y and Steitz JA (2007). Switching from repression to activation: microRNAs can up-regulate translation. Science 318: 1931-1934.

Vinci S, Gelmini S, Pratesi N, Conti S, et al. (2011). Genetic variants in miR-146a, miR-149, miR-196a2, miR-499 and their influence on relative expression in lung cancers. Clin. Chem. Lab. Med. 49: 2073-2080.

Xiang Y, Fan S, Cao J, Huang S, et al. (2012). Association of the microRNA-499 variants with susceptibility to hepatocellular carcinoma in a Chinese population. Mol. Biol. Rep. 39: 7019-7023.

Zeng Y and Cullen BR (2003). Sequence requirements for micro RNA processing and function in human cells. RNA 9: 112-123.

Zhou B, Wang K, Wang Y, Xi M, et al. (2011). Common genetic polymorphisms in pre-microRNAs and risk of cervical squamous cell carcinoma. Mol. Carcinog. 50: 499-505.

Zhou J, Lv R, Song X, Li D, et al. (2012). Association between two genetic variants in miRNA and primary liver cancer risk in the Chinese population. DNA Cell Biol. 31: 524-530. 